University of Wollongong

Research Online

Faculty of Engineering and Information

Faculty of Engineering and Information

Sciences - Papers: Part B

Sciences

2018

Changes in gait and plantar foot loading upon using vibrotactile wearable biofeedback system in patients with stroke

Christina Ma

The Hong Kong Polytechnic University

Yong-Ping Zheng

The Hong Kong Polytechnic University

Winson Lee

University of Wollongong, ccwlee@uow.edu.au

Follow this and additional works at: https://ro.uow.edu.au/eispapers1

Part of the Engineering Commons, and the Science and Technology Studies Commons

Research Online is the open access institutional repository for the University of Wollongong. For further information contact the UOW Library: research-pubs@uow.edu.au 


\title{
Changes in gait and plantar foot loading upon using vibrotactile wearable biofeedback system in patients with stroke
}

\author{
Abstract \\ Background: Patients with stroke walk with excessive foot inversion at the affected side, which may \\ disturb their balance and gait. Objectives: This study aimed to investigate the effects of instant \\ biofeedback of plantar force at the medial and lateral forefoot regions on gait and plantar foot loading in \\ patients with stroke. Methods: A total of eight patients with hemiplegic stroke, who had flexible rearfoot \\ varus deformity at the affected side, participated in this study. A vibrotactile biofeedback system was \\ developed and evaluated. It analyzed forces at the medial and lateral forefeet, and instantly provided \\ vibration clues when the plantar force at medial forefoot was less than a threshold. Each subject's three- \\ dimensional gait parameters and plantar-pressure distribution during walking were measured under two \\ experimental conditions (sequence randomized): with and without the device turned on (Trial-registration \\ number: ChiCTR-IPB-15006530 and HKCTR-1853). Results: Providing biofeedback significantly reduced \\ the foot inversion and increased the mid-stance foot-floor contact area and medial midfoot plantar \\ pressure of the affected limb, bringing the values of these parameters closer to those of the unaffected \\ side. The biofeedback also significantly reduced the unaffected side's excessive knee flexion and hip \\ abduction. Conclusions: There were signs of improved foot loading characteristics and gait upon \\ provision of instant vibrotactile biofeedback of plantar force. The positive results of this study further \\ support the development of wearable biofeedback devices for improving gait of patients with stroke.

\section{Disciplines} \\ Engineering | Science and Technology Studies

\section{Publication Details} \\ Ma, C., Zheng, Y. \& Lee, W. (2018). Changes in gait and plantar foot loading upon using vibrotactile \\ wearable biofeedback system in patients with stroke. Topics in Stroke Rehabilitation, 25 (1), 20-27.
}




\title{
1 Changes in Gait and Plantar Foot Loading upon Using \\ 2 Vibrotactile Wearable Biofeedback System in Patients with \\ 3 Stroke
}

\begin{abstract}
Background: Patients with stroke walk with excessive foot inversion at the affected side, which may disturb their balance and gait. Objectives: This study aimed to investigate the effects of instant biofeedback of plantar force at the medial and lateral forefoot regions on gait and plantar foot loading in patients with stroke. Methods: A total of eight patients with hemiplegic stroke, who had flexible rearfoot varus deformity at the affected side, participated in this study. A vibrotactile biofeedback system was developed and evaluated. It analysed forces at the medial and lateral forefeet, and instantly provided vibration clues when the plantar force at medial forefoot was less than a threshold. Each subject's threedimensional gait parameters and plantar-pressure distribution during walking were measured under two experimental conditions (sequence randomized): with and without the device turned-on (Trial-registration number: ChiCTR-IPB15006530\& HKCTR-1853). Results: Providing biofeedback significantly reduced the foot inversion and increased the mid-stance foot-floor contact area and medial midfoot plantar pressure of the affected limb, bringing the values of these parameters closer to those of the unaffected-side. The biofeedback also significantly reduced the unaffected-side's excessive knee flexion and hip abduction. Conclusions: There were signs of improved foot loading characteristics and gait upon provision of instant vibrotactile biofeedback of plantar force. The positive results of this study further support the development of wearable biofeedback devices for improving gait of patients with stroke.
\end{abstract}

Keywords: stroke; foot inversion; plantar pressure; instant biofeedback; gait training; smart wearable device

\section{Introduction}

Stroke is a leading cause of neurological impairment [1] and chronic motor disability

[2] in adults. Motor impairments of lower limbs can lead to difficulty in locomotion and 
activities of daily living, and consequently influence an individual's quality of life [3].

32 People with stroke generally walk with higher gait asymmetry [4], energy consumption

33 [5] and risk of fall [6]. Abnormal motion of the ankle-foot complex contributes to the deterioration of the overall balance performance and gait pattern [7]. Deformities at the ankle joint are common, due to the muscle spasticity [8] and muscle imbalance [9]. The foot at the affected side of people with stroke tends to be more plantar-flexed and inverted than people without stroke [10]. Recovery of walking ability by addressing the ankle-joint deformity helps patients with stroke to regain the independence in daily life, and is one of the main rehabilitation training goals [11]. Plantarflexion deformity can increase the chance of fall, as the feet tend to drag over the floor during swing phase [12]. Fortunately, ankle-foot orthoses have been used successfully to correct plantar-flexion deformity after stroke [13]. Correcting varus deformity has been more difficult, because of the lack of lever arm that provides sufficient corrective eversion moment at foot. Abnormally high degree of foot inversion during gait could put excessively more strains on muscles and tendons [14] and more 46 plantar forces at the lateral side of paretic foot [15]. Such musculoskeletal overloading could lead to soft tissue damage and structural deformity at the foot, leading to foot pain

48 [16]. Foot inversion also reduces the total contact area with ground during mid-stance and the propulsive force during push-off phases of the gait [17]. Foot pain together with

50 the altered foot biomechanics could disturb gait and consequently predispose the

51 individuals with higher risk of falls [18]. Previous studies have concluded that increased

52 foot inversion is associated with decreased postural stability [19, 20], which is a crucial

53 indicator of increased risk of falls [21]. Reducing the degree of abnormal foot inversion

54 is required to relieve muscle stress and foot pain, which could improve walking performance and reduce risk of falls in patients with stroke [14]. 
Various interventions have been used to relieve varus deformity for patients with

57 stroke, but with some limitations [9]. Local botulinum toxin injection has the limitations

of high cost and transient nature that requires repetitive injections [22]. Patient's

compliance of wearing ankle-foot orthosis has been low, thus leading to a high financial

loss for society and a waste of therapeutic effort as reviewed in [23]. Physiotherapy

which provides repetitive verbal reminders of putting the foot in a better position during gait requires intensive manpower [24].

Wearable biofeedback devices have great potential of facilitating home-based

trainings in patients, which contribute to high level of continuity, adherence, and compliance rates of training in patients [25] and save the expertise human resources.

Biofeedback devices, with the use of sensors (force sensors, accelerometers, gyroscopes and magnetometers) and feedback modalities (screens, speakers and vibrators) [26], were used in the elderly [27-29], healthy young adults [28-31], patients with vestibular disease [27, 32], patients with Parkinson's disease [33], and lower limb amputees [34, 35]. Regarding stroke patients, researchers have detected stance time using foot switches [36, 37], ground reaction forces using force sensors [33] and body sway using smartphones [38] and inertial motion sensors [33]. Upon giving some instant feedback based on the sensor measurements, some improvements in the amount of body sway

$74[30,35]$, symmetries in weight-bearing and stance/swing time between two legs [33, 34], as well as scores in standard clinical tests were noted [30]. However, there is a lack of comprehensive understanding on how biofeedback devices could influence the spatial-temporal, kinetic and kinematic gait parameters of stroke patients. In addition, little attempt has been made to address the negative effects of varus deformities on gait through biofeedback. 
This paper aims to: (1) present a biofeedback system that reminds stroke patients with flexible foot varus deformity to increase loading at the medial aspect of the foot of the affected side during gait; and (2) report the effects of using such biofeedback system on gait parameters and plantar pressure distribution. It is hypothesized that instant

84 vibrotactile biofeedback of plantar force at the medial and lateral forefoot could

85 improve plantar loading at the medial aspect of the affected foot and the gait pattern of 86 stroke patients with flexible foot varus deformity.

\section{Materials and Methods}

\section{$88 \quad$ Subjects}

89 Convenience sampling approach was used to recruit eight hemiplegic patients (seven 90 males and one female) with an average age of 53.5 years, in this study (table 1 ). The causes of the stroke in these patients were ischemic in six and haemorrhage in two patients. The average duration since the onset of stroke was 3.8 years. Two subjects were hemiplegic at the left sides and the remaining six were at the right sides. All subjects were referred by a local Physiotherapy Clinic where they received trainings for treating dynamic balance disorder. They were unilateral hemiplegia caused by cerebral hemisphere stroke, living in a community-based setting, able to walk independently without walking assisting devices for more than 10 meters, and with good cooperation and compliance in gait analysis. All subjects were able to understand and follow the experimental instructions. They did not have fixed deformities over the ankle joint complex, but had rearfoot varus deformity at the affected side which could be corrected by external corrective forces, as evaluated by a Certified Orthotist following standard procedures specified in [39]. Subjects who had other peripheral or central nervous system dysfunctions, active inflammatory or pathologic changes in the joints of lower 
104 extremities in the previous 6 months, and active medical problems were not included in

105 this study. All subjects have signed written-informed consents before participating in

106 the study. Ethical approval was granted from the Human Subjects Ethics Sub-committee

107 of The Hong Kong Polytechnic University (HSEARS20140211002). This study was

108 registered on the Chinese Clinical Trial Registry (ChiCTR-IPB-15006530) and the

109 Hong Kong Clinical Trial Registry (HKCTR-1853).

\section{The Biofeedback System}

111 The vibrotactile biofeedback system consisted of two separate components of 1) a

112 plantar force acquisition unit $(5.5 \mathrm{~cm} \times 2.5 \mathrm{~cm} \times 1.7 \mathrm{~cm})$ and 2$)$ a vibration feedback unit

$113(4.5 \mathrm{~cm} \times 2.2 \mathrm{~cm} \times 1.5 \mathrm{~cm})$ that were both attached to the subjects' affected side (figure 1$)$.

114 The plantar force acquisition unit consisted of two thin-film force sensors (A301,

115 Tekscan Co., Ltd, USA), a microprocessor unit (ATMEGA328P, Atmel Co., Ltd,

116 USA), a wireless transmitter module (HC-05, HC information Tech. Co., Ltd, China),

117 and a rechargeable lithium-ion battery (FLB-16340-880-PTD, UltraFire Co., Ltd,

118 China). The vibration feedback unit consisted of one vibrator (XY-B1027-DX,

119 Xiongying electronics Co., Ltd, China), a wireless receiver module (HC-05, HC

120 information Tech. Co., Ltd, China), and a rechargeable lithium-ion battery (FLB-16340-

121 880-PTD, UltraFire Co., Ltd, China).

122 The two thin-film force sensors $(25.4 \mathrm{~mm} \times 14 \mathrm{~mm} \times 0.203 \mathrm{~mm}$, sensing area

$1239.53 \mathrm{~mm}$ diameter each) were attached by adhesive tapes to the bottom of a piece of

124 2mm-thick flat insole, which was made of a medium firm (30-35 Shore A Hardness)

125 ethylene-vinyl acetate (EVA, Foot Specialist Footcare \& Products Co. Ltd, HK). The

126 sensors were located at the first and fifth metatarsal heads of the affected side, verified

127 by a certified orthotist, to evaluate the medial and lateral plantar force. One vibrator 
128 (10mm diameter $\times 2.7 \mathrm{~mm}$ height) was fastened by an elastic strap at the subject's wrist

129 of the affected side. The vibrator was set to produce full magnitude of vibration when

130 the real-time forces measured at the first metatarsal head was less than $50 \%$ of that

131 measured at the fifth metatarsal head at the same walking step. The vibrator was not

132 activated in other conditions. Pilot studies showed that other ratios (25\% and $100 \%)$ did

133 not appear to provide appropriate reminder on foot inversion to subjects.

134 The plantar force acquisition unit analysed the force data at foot soles and

135 delivered control signals to the vibration feedback unit via Bluetooth communication.

136 The vibration frequency and strength of the vibrator were $220 \mathrm{~Hz}$ and $1 \mathrm{G}$, respectively,

137 which were found to be highly recognizable by humans [40]. All subjects were assessed

138 before the experiment to ensure that they could perceive the vibration of the vibrators.

139 Both sampling frequency and transmission rate of the device were $10 \mathrm{~Hz}$. The

140 rechargeable batteries enabled the entire system to function for 24 hours continuously.

141 The entire biofeedback system weighed less than 70 grams.

\section{Experimental Design and Procedures}

143 This study was conducted in a university locomotion laboratory. All subjects were

144 explained how the biofeedback system functioned prior to the experiment. They were

145 informed that the vibration of the vibrator corresponded to the excessive foot inversion

146 at the affected lower limb. They were instructed to put more loading at the medial

147 forefoot when the vibrator was activated. During the practicing period, the subjects

148 were instructed to shift weight between the medial and lateral foot and experience the

149 vibrations, to ensure that they understood the function of this system and were capable

150 of using the feedback vibrations as a training aid. Subjects were given 10 minutes to get

151 familiar with the new biofeedback system [41]. 

instructed to walk along a smooth, horizontal 7m-long walkway at a comfortable speed.

154 The sequence of two testing conditions was randomly assigned to each subject: 1) with

155 the biofeedback system turned-off; and 2) with the biofeedback system turned-on.

156 Subjects were blinded from the experimental condition during the experiment. Same

157 instructions were given to the subjects as to the actions they should take when there was

158 a vibration feedback. Each testing condition was repeated 5 times consecutively for 159 each subject. Between two conditions, each subject was given a 10-minute rest to

160 eliminate the possible effect of fatigue. If subjects verbally reported any kinds of

161 discomfort during the experiment, the experiment would be stopped with the situation

162 being recorded. Two complete gait cycles in the middle of each walking trial

163 (containing a total of 7-9 walking steps) were extracted to avoid the variable steps 164 associated with initiation and termination of gait [42]. This strategy also enabled to 165 collect data of one full gait cycle for both affected and unaffected sides, as well as the 166 sufficient number of strides that are required to achieve high reliability when analysing

167 gait parameters [43]. During the experiment, all subjects wore the same shoe model 168 (TFGF81722/TFGF82722, TOREAD ${ }^{\circledR}$, TOREAD Co., Ltd, China) provided by the 169 researchers.

\section{Outcome Measures}

171 An in-shoe plantar pressure measurement system (novel pedar-x system, Pedar ${ }^{\mathrm{TM}}$, novel

$172 \mathrm{GmbH}$, Munich, DE), which was shown to have high repeatability [44] and validity

173 [45], was sampling at $50 \mathrm{~Hz}$ and used to measure the plantar pressure distribution

174 during walking in 2 experimental conditions. Before and after data collection of each

175 subject, the insoles were checked using the Trublu ${ }^{\circledR}$ calibrating system to ascertain that 
176 all sensors produced accurate and reproducible absolute values [46]. The plantar foot

177 was divided into six regions: medial forefoot, lateral forefoot, medial midfoot, lateral

178 midfoot, medial rearfoot, and lateral rearfoot (figure 2). For all subjects, the forefoot,

179 midfoot, and rearfoot regions comprised the first 35\%, the following 35\%, and the

180 remaining $30 \%$ of the foot length, respectively.

181 An eight-camera three-dimensional (3D) motion capture system (Vicon Nexus

182 1.8.1, Vicon Nexus ${ }^{\mathrm{TM}}$, Vicon Motion Systems Ltd., UK), sampling at $100 \mathrm{~Hz}$, was used

183 to measure the 3D kinetic data in subjects during over-ground walking in 2

184 experimental conditions. A built-in lower limb marker set (Plug-in Gait Model) was

185 adopted, in which 15 infra-red reflective markers were affixed to both sides at the heels,

186 foot dorsum, lateral malleolus, lateral femoral condyles, middle of thighs/shanks,

187 anterior superior iliac spines, and iliac crest. Spatial-temporal and kinematic data were

188 measured and analysed using the Plug-in Gait Model in Vicon system. The gait data

189 were low-pass filtered using a $4^{\text {th }}$ order Butterworth filter with a $6 \mathrm{~Hz}$ cut-off frequency.

\section{Statistical Analysis}

191 The parameters included for analysis were the average and peak plantar pressure

192 parameters at each of the six plantar foot regions, total foot-floor contact area, stance

193 time, swing time, stride time, walking speed, and peak lower limb joint angles during

194 both stance and swing phases. Statistical analysis was performed using Statistical

195 Package for Social Sciences (SPSS, version 22.0, IBM Corporation, Armonk, NY,

196 USA). Two-way repeated measures ANOVA was performed prior to examine the main

197 effect of "interventions" (with vs. without biofeedback), the main effect of "limbs"

198 (affected vs. unaffected side), and the interaction effect between two variables

199 (interventions $\times$ limbs) in all measured parameters among all subjects. If significant 
200 interaction effect was found in ANOVA, pair-wise comparisons of "interventions" (with

201 vs. without biofeedback) and "limbs" (affected vs. unaffected limb) were performed by

202 using t-tests with Bonferroni corrections. The level of significance was set at 0.05.

203 During data analysis, the person who analysed data did not know the content of each

204 test condition, as conditions were coded.

\section{Results}

206 None of the subjects verbally reported any discomfort related to the use of the

207 biofeedback during the experiment. The following shows the significant changes in gait

208 variables and plantar pressure distribution upon using the biofeedback.

\section{Changes in kinematic variables}

210 Without the biofeedback, the peak foot inversion of the affected side during swing

211 phase (angle 25.1 degrees) was 39.1\%-significantly more than the unaffected side

212 ( $\mathrm{p}=0.047)$. Turning on the biofeedback system led to a significant $17.2 \%$ reduction of

213 peak foot inversion ( $\mathrm{p}=0.012)$ at the affected limb during swing phase (angle 20.8

214 degrees) (figure 3).

215 When the biofeedback system was turned off, the unaffected side had

216 significantly more peak knee flexion $(\mathrm{p}=0.047)$ during swing phase and more peak hip

217 abduction during both stance $(\mathrm{p}=0.024)$ and swing $(\mathrm{p}=0.075)$ phases than the affected

218 side. Turning on the biofeedback device significantly reduced the unaffected-side peak

219 knee flexion during swing phase $(\mathrm{p}=0.009)$ and peak hip abduction during stance phase

220 ( $\mathrm{p}=0.017)$. There was no longer significant difference in peak hip abductions between

221 the 2 legs after turning on the device (figure 3). 
223 With the biofeedback system turned off, the total foot-floor contact area in mid-stance

224 phase $(\mathrm{p}=0.040)$ and the peak plantar pressure at the medial midfoot $(\mathrm{p}=0.034)$ of the

225 affected limb were significantly lower than those of the unaffected limb. When it was

226 turned on, such contact area $(\mathrm{p}=0.001)$ and plantar pressure $(\mathrm{p}=0.001)$ at the affected

227 limb were then significantly increased. There was no longer significant difference in

228 total foot-floor contact area or peak plantar pressure at the medial midfoot between the

2292 legs after turning on the device (figure 4\&5).

230 Changes in kinematic variables and plantar-pressure distribution that happened 231 at both the affected and unaffected sides

232 While turning on the biofeedback device did not significantly change the walking speeds, it significantly increased the stance $(\mathrm{p}=0.003)$ and stride $(\mathrm{p}=0.001)$ time, average plantar pressure at medial forefoot $(\mathrm{p}=0.001)$, peak $(\mathrm{p}=0.001)$ and average ( $p=0.020$ ) plantar pressure at medial midfoot of both limbs (figure 4\&5).

\section{Discussion}

This study developed a plantar-force based vibrotactile biofeedback and investigated the effects of its use on plantar foot loading and gait in hemiplegic stroke patients with flexible foot varus deformity. With no biofeedback, the foot inversion angle at the affected side was significantly higher than the unaffected side. The biofeedback device attempted to relieve foot varus by giving vibration clues when the medial side of the affected foot did not exert high enough forces during walking. This significantly reduced the maximum foot inversion of the affected side during swing phase.

244 Significant increase in the plantar force at the medial forefoot during stance phase and

245 total foot-floor contact area were then observed. This potentially improves postural 
balance [47], reduces chances of developing foot pain [14], and soft tissue injury [16].

It is interesting to note that while the device provided feedback on the weight bearing characteristics of the foot at the affected side, significant changes were observed at the unaffected side. Without turning-on the biofeedback device, subjects walked with significantly more peak hip abduction and knee flexion during swing phase at the unaffected side than the affected side, and these angles were higher than people without stroke [17]. Increasing hip abduction widened the base of support, which might compensate for the reduced walking balance caused by the abnormal orientation and loading of the feet at the affected side [17, 48]. Meanwhile, excessive knee flexion provides more foot clearance during swing phase at which the entire body weight is put against the opposite limb $[17,49,50]$. Turning on the device significantly reduced the unaffected-side knee flexion during swing phase and hip abduction during stance phases. Such reductions decreased the asymmetry between affected and unaffected legs. The improved symmetry of hip and knee joints during walking could improve the walking efficiency of patients of stroke [51].

The stance time of both limbs increased while walking speed did not have significant changes upon using the biofeedback device. The significantly increased stance time could reflect that subjects were more confident of bearing weight on their feet [52], indicating better walking capacity [53]. The biofeedback device did not compromise walking speed. This suggested that subjects did not need to walk more carefully and slowly when paying attention to the reminder signals from the device,

267 which is consistent with a previous study identified retained beneficial effects of 268 vibrotactile biofeedback when subjects performed dual cognitive tasks while receiving

269 vibrotactile stimulations [54]. This also indicates that the changes in plantar pressure 270 were not due to variations in walking speed. 
In this study, the threshold ratio of provoking vibrotactile feedback was set at a

272 level at which the plantar force at the medial forefoot reached $50 \%$ of that at the lateral

273 forefoot. The threshold was chosen from a series of threshold ratios in pilot study,

274 including 25\%, 50\% and 100\%. It appeared that the ratio of $25 \%$ was too easy for the

275 subjects to achieve, which lowered the value of using the device for gait training; while

276 the ratio of $100 \%$ was too difficult for subjects to achieve in a limited training time

277 period, leading to unstopped vibrations during walking. Subjects cannot benefit from

278 the unstopped vibration, as no useful differentiated reminders were provided. It is

279 worthwhile to involve more threshold ratios and further explore the best setting of the

280 device in the future.

281 The clinical implication of this study is that a device measuring plantar forces

282 and providing instant biofeedback has great potentials of improving gait in people with

283 stroke. Subjects did not verbally report any discomfort upon using the biofeedback

284 device in this study. Embedding thin-film force sensors into shoes/insoles and using

285 appropriate feedback devices facilitate realization of home-based rehabilitation

286 programs, which have high level of continuity, adherence, and compliance rates of

287 training in patients $[25,55]$. The nature of low interference with daily tasks of

288 vibrotactile biofeedback [27] also allows the device to be used as a walking aid, which

289 is capable of continuously monitoring the foot posture and walking ability, in both

290 indoor and outdoor daily activities in the future.

291 This study investigated the immediate effect of this wearable vibrotactile

292 biofeedback device on plantar loading and gait pattern in patients with chronic stroke.

293 Future study shall investigate if such positive effects retained after long-term use, and in

294 home-based settings. The evaluation of the applicability and repeatability of the device

295 could be conducted in the future. The sample size of this study was rather small, while 
there are also some other published papers with small sample size demonstrated an

297 effect of biofeedback devices [36, 56-59]. Future studies shall investigate the effect of such plantar force-based biofeedback device in larger samples who have poor walking ability.

\section{Conclusions}

301 Subjects in this study showed significant improvements in foot loading and gait upon using instant vibrotactile biofeedback regarding medical and lateral forefoot loadings. Instant vibrotactile biofeedback of plantar force at the medial and lateral forefoot significantly reduced the abnormally excessive foot inversion angle by more than $15 \%$. This significantly increased foot-floor contact area and weight bearing over the medial aspect of the foot of the affected limb, which might help improve balance and walking capability. Improvements in gait patterns were also noted as the biofeedback significantly reduced the excessive hip abduction and knee flexion of the unaffected

309 limb.

\section{Acknowledgements}

311 This work was supported by [The Hong Kong Polytechnic University Research Studentship];

312 under Grant [number: RTNR]; the [Innovation and Technology Commission of Hong Kong

313 SAR]; under Grant [number: ITS/030/13]; and the [Institute of Active Aging, The Hong Kong

314 Polytechnic University].

\section{Declaration of interest}

316 The authors report no conflicts of interest.

\section{References}

318 1. Whitall, J., Stroke rehabilitation research: time to answer more specific

319 questions? Neurorehabilitation and neural repair 2004, 18, (1), 3-8.

$320 \quad 2$. Bath, P.; Bath, F., Prostacyclin and analogues for acute ischaemic stroke.

$321 \quad$ Cochrane Database Syst Rev 2004, 3. 
3. Kim, K.; Kim, Y. M.; Kim, E. K., Correlation between the activities of daily 
21. Moghadam, M.; Ashayeri, H.; Salavati, M.; Sarafzadeh, J.; Taghipoor, K. D.; Saeedi, A.; Salehi, R., Reliability of center of pressure measures of postural stability in healthy older adults: effects of postural task difficulty and cognitive load. Gait \& posture 2011, 33, (4), 651-655.

22. Ozcakir, S.; Sivrioglu, K., Botulinum toxin in poststroke spasticity. Clinical medicine \& research 2007, 5, (2), 132-138.

23. Swinnen, E.; Kerckhofs, E., Compliance of patients wearing an orthotic device or orthopedic shoes: A systematic review. Journal of bodywork and movement therapies 2015, 19, (4), 759-770.

24. Hesse, S., Rehabilitation of gait after stroke: evaluation, principles of therapy, novel treatment approaches, and assistive devices. Topics in Geriatric Rehabilitation 2003, 19, (2), 109-126.

25. Davis, J. C.; Robertson, M. C.; Ashe, M. C.; Liu-Ambrose, T.; Khan, K. M.; Marra, C. A., Does a home based strength and balance programme in people aged $\geqslant 80$ years provide the best value for money to prevent falls?: A systematic review of economic analyses of falls prevention interventions. British journal of sports medicine 2009.

26. Ma, C. Z.-H.; Wong, D. W.-C.; Lam, W. K.; Wan, A. H.-P.; Lee, W. C.-C., Balance improvement effects of biofeedback systems with state-of-the-art wearable sensors: a systematic review. Sensors 2016, 16, (4), 434.

27. Wall III, C., Application of vibrotactile feedback of body motion to improve rehabilitation in individuals with imbalance. Journal of neurologic physical therapy: JNPT 2010, 34, (2), 98-104.

28. Ma, C. Z.-H.; Wan, A. H.-P.; Wong, D. W.-C.; Zheng, Y.-P.; Lee, W. C.-C., A Vibrotactile and Plantar Force Measurement-Based Biofeedback System: Paving the Way towards Wearable Balance-Improving Devices. Sensors 2015, 15, (12), 31709-31722.

29. Ma, C. Z.; Wan, A. H.; Wong, D. W.; Zheng, Y.-P.; Lee, W. C. In Improving postural control using a portable plantar pressure-based vibrotactile biofeedback system, Biomedical Engineering and Sciences (IECBES), 2014 IEEE Conference on, 2014; IEEE: 2014; pp 855-860.

30. Vuillerme, N.; Cuisinier, R., Head position-based electrotactile tongue biofeedback affects postural responses to Achilles tendon vibration in humans. Exp Brain Res 2008, 186, (3), 503-8.

31. Ma, C. Z.-H.; Lee, W. C.-C., A wearable vibrotactile biofeedback system improves balance control of healthy young adults following perturbations from quiet stance. Human Movement Science 2017, 55, 54-60.

32. Sienko, K. H.; Balkwill, M. D.; Wall, C., 3rd, Biofeedback improves postural control recovery from multi-axis discrete perturbations. J Neuroeng Rehabil 2012, 9, 53.

33. Byl, N.; Zhang, W.; Coo, S.; Tomizuka, M., Clinical impact of gait training enhanced with visual kinematic biofeedback: Patients with Parkinson's disease and patients stable post stroke. Neuropsychologia 2015.

34. Lee, M. Y.; Lin, C. F.; Soon, K. S., Balance control enhancement using subsensory stimulation and visual-auditory biofeedback strategies for amputee subjects. Prosthet Orthot Int 2007, 31, (4), 342-52.

35. Wan, A. H.; Wong, D. W.; Ma, C. Z.; Zhang, M.; Lee, W. C., Wearable vibrotactile biofeedback device allowing identification of different floor conditions for lower-limb amputees. Archives of physical medicine and rehabilitation 2016. 
36. Afzal, M. R.; Byun, H. Y.; Oh, M. K.; Yoon, J., Effects of kinesthetic haptic feedback on standing stability of young healthy subjects and stroke patients. $J$ Neuroeng Rehabil 2015, 12, 27.

37. Sungkarat, S.; Fisher, B. E.; Kovindha, A., Efficacy of an insole shoe wedge and augmented pressure sensor for gait training in individuals with stroke: a randomized controlled trial. Clinical rehabilitation 2011, 25, (4), 360-369.

38. Afzal, M. R.; Oh, M.-K.; Lee, C.-H.; Park, Y. S.; Yoon, J., A Portable Gait Asymmetry Rehabilitation System for Individuals with Stroke Using a Vibrotactile Feedback. BioMed Research International 2015.

39. Magee, D. J., Orthopedic Physical Assessment-E-Book. Elsevier Health Sciences: 2014.

40. $\quad$ Kyung, K.-U.; Ahn, M.; Kwon, D.-S.; Srinivasan, M. In Perceptual and biomechanical frequency response of human skin: implication for design of tactile displays, Eurohaptics Conference, 2005 and Symposium on Haptic Interfaces for Virtual Environment and Teleoperator Systems, 2005. World Haptics 2005. First Joint, 2005; IEEE: 2005; pp 96-101.

41. Boonsinsukh, R.; Panichareon, L.; Saengsirisuwan, V.; Phansuwan-Pujito, P., Clinical identification for the use of light touch cues with a cane in gait rehabilitation poststroke. Topics in stroke rehabilitation 2011, 18, (sup1), 633642.

42. Chen, C.; Hong, P.; Chen, C.; Chou, S. W.; Wu, C.; Cheng, P.; Tang, F.; Chen, $\mathrm{H}$., Ground reaction force patterns in stroke patients with various degrees of motor recovery determined by plantar dynamic analysis. Chang Gung medical journal 2007, 30, (1), 62.

43. Hollman, J. H.; Childs, K. B.; McNeil, M. L.; Mueller, A. C.; Quilter, C. M.; Youdas, J. W., Number of strides required for reliable measurements of pace, rhythm and variability parameters of gait during normal and dual task walking in older individuals. Gait \& posture 2010, 32, (1), 23-28.

44. Putti, A.; Arnold, G.; Cochrane, L.; Abboud, R., The Pedar ${ }^{\circledR}$ in-shoe system: Repeatability and normal pressure values. Gait \& posture 2007, 25, (3), 401405.

45. Price, C.; Parker, D.; Nester, C., Validity and repeatability of three in-shoe pressure measurement systems. Gait \& posture 2016, 46, 69-74.

46. Ramanathan, A.; Kiran, P.; Arnold, G.; Wang, W.; Abboud, R., Repeatability of the Pedar- $\mathrm{X}{ }^{\circledR}$ in-shoe pressure measuring system. Foot and Ankle Surgery 2010, 16, (2), 70-73.

47. Hertel, J.; Gay, M. R.; Denegar, C. R., Differences in postural control during single-leg stance among healthy individuals with different foot types. Journal of athletic training 2002, 37, (2), 129.

48. Reinbolt, J. A.; Fox, M. D.; Arnold, A. S.; Õunpuu, S.; Delp, S. L., Importance of preswing rectus femoris activity in stiff-knee gait. Journal of Biomechanics 2008, 41, (11), 2362-2369.

49. Mills, P. M.; Barrett, R. S.; Morrison, S., Toe clearance variability during walking in young and elderly men. Gait \& posture 2008, 28, (1), 101-107.

50. Woolley, S. M., Characteristics of gait in hemiplegia. Topics in stroke rehabilitation 2015.

51. Brouwer, B.; Parvataneni, K.; Olney, S. J., A comparison of gait biomechanics and metabolic requirements of overground and treadmill walking in people with stroke. Clinical Biomechanics 2009, 24, (9), 729-734. 
52. Mâaref, K.; Martinet, N.; Grumillier, C.; Ghannouchi, S.; André, J. M.; Paysant, J., Kinematics in the terminal swing phase of unilateral transfemoral amputees: microprocessor-controlled versus swing-phase control prosthetic knees. Archives of physical medicine and rehabilitation 2010, 91, (6), 919-925.

53. Jonkers, I.; Delp, S.; Patten, C., Capacity to increase walking speed is limited by impaired hip and ankle power generation in lower functioning persons poststroke. Gait \& posture 2009, 29, (1), 129-137.

54. Haggerty, S.; Jiang, L.-T.; Galecki, A.; Sienko, K. H., Effects of biofeedback on secondary-task response time and postural stability in older adults. Gait \& posture 2012, 35, (4), 523-528.

55. Madureira, M. M.; Takayama, L.; Gallinaro, A.; Caparbo, V.; Costa, R.; Pereira, R. M., Balance training program is highly effective in improving functional status and reducing the risk of falls in elderly women with osteoporosis: a randomized controlled trial. Osteoporosis International 2007, 18, (4), 419-425.

56. Crea, S.; Cipriani, C.; Donati, M.; Carrozza, M. C.; Vitiello, N., Providing timediscrete gait information by wearable feedback apparatus for lower-limb amputees: usability and functional validation. Neural Systems and Rehabilitation Engineering, IEEE Transactions on 2015, 23, (2), 250-257.

57. Wall III, C.; Weinberg, M. S.; Schmidt, P. B.; Krebs, D. E., Balance prosthesis based on micromechanical sensors using vibrotactile feedback of tilt. Biomedical Engineering, IEEE Transactions on 2001, 48, (10), 1153-1161.

58. Lee, M.-Y.; Lin, C.-F.; Soon, K.-S., Balance control enhancement using subsensory stimulation and visual-auditory biofeedback strategies for amputee subjects. Prosthet. Orthot. Int. 2007, 31, (4), 342-352.

59. Alahakone, A. U.; Senanayake, S.; Arosha, M., A real-time system with assistive feedback for postural control in rehabilitation. Mechatronics, IEEE/ASME Transactions on 2010, 15, (2), 226-233. 
501 Table 1. Subject information

\begin{tabular}{|cccccccc|}
\hline Subject & $\begin{array}{c}\text { Age } \\
\text { (years) }\end{array}$ & Gender & $\begin{array}{c}\text { Weight } \\
(\mathbf{k g})\end{array}$ & $\begin{array}{c}\text { Height } \\
(\mathbf{m})\end{array}$ & $\begin{array}{c}\text { Causes of } \\
\text { stroke }\end{array}$ & $\begin{array}{c}\text { Duration } \\
\text { after } \\
\text { stroke } \\
\text { (years) }\end{array}$ & $\begin{array}{c}\text { Hemiplegic } \\
\text { side }\end{array}$ \\
\hline 1 & 68 & $\mathrm{~F}$ & 54.5 & 1.63 & Ischemic & 3 & $\mathrm{~L}$ \\
\hline 2 & 50 & $\mathrm{M}$ & 73.5 & 1.78 & Ischemic & 14 & $\mathrm{R}$ \\
\hline 3 & 50 & $\mathrm{M}$ & 61.5 & 1.81 & Ischemic & 1 & $\mathrm{R}$ \\
\hline 4 & 58 & $\mathrm{M}$ & 70.0 & 1.80 & Hemorrhage & 3 & $\mathrm{R}$ \\
\hline 5 & 47 & $\mathrm{M}$ & 74.0 & 1.75 & Ischemic & 1 & $\mathrm{~L}$ \\
\hline 6 & 67 & $\mathrm{M}$ & 87.0 & 1.78 & Ischemic & 2 & $\mathrm{R}$ \\
\hline 7 & 41 & $\mathrm{M}$ & 85.0 & 1.75 & Hemorrhage & 4 & $\mathrm{R}$ \\
\hline 8 & 47 & $\mathrm{M}$ & 73.5 & 1.71 & Ischemic & 2 & $\mathrm{R}$ \\
\hline
\end{tabular}




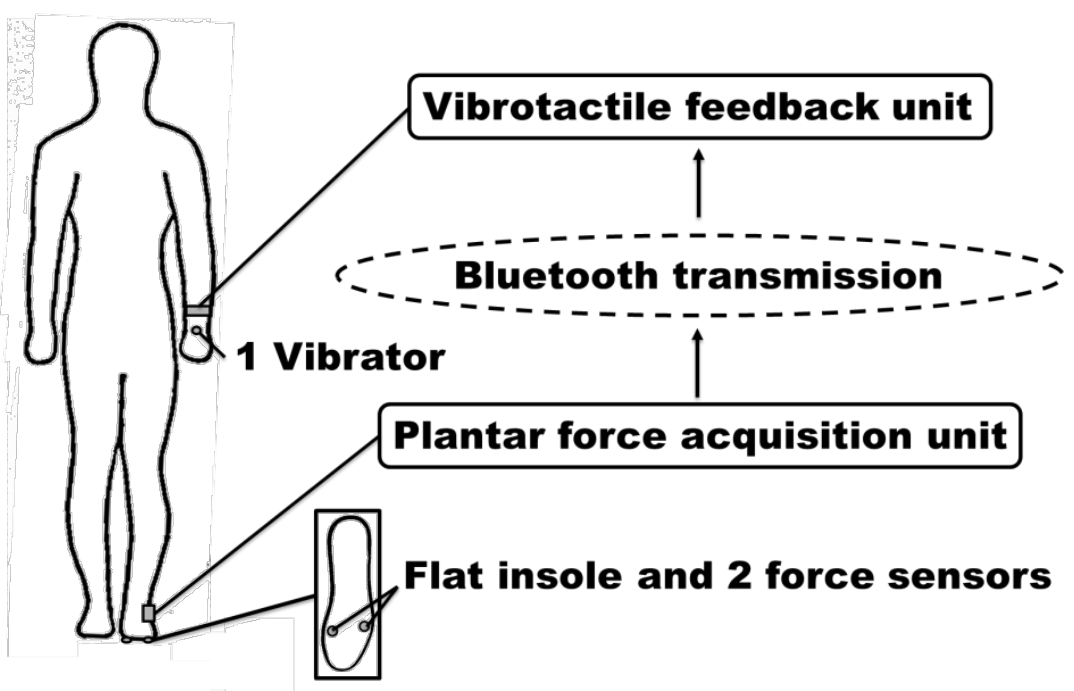

504 Figure 1. The vibrotactile system, consisted of a plantar force

505 acquisition unit and a vibrotactile feedback unit wirelessly connected 
506

508

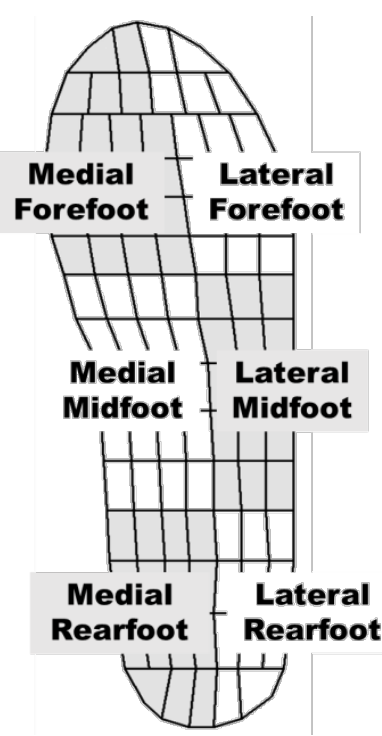

509 Figure 2. Foot regions: medial forefoot, lateral forefoot, medial

510 midfoot, lateral midfoot, medial rearfoot, and lateral rearfoot 
511
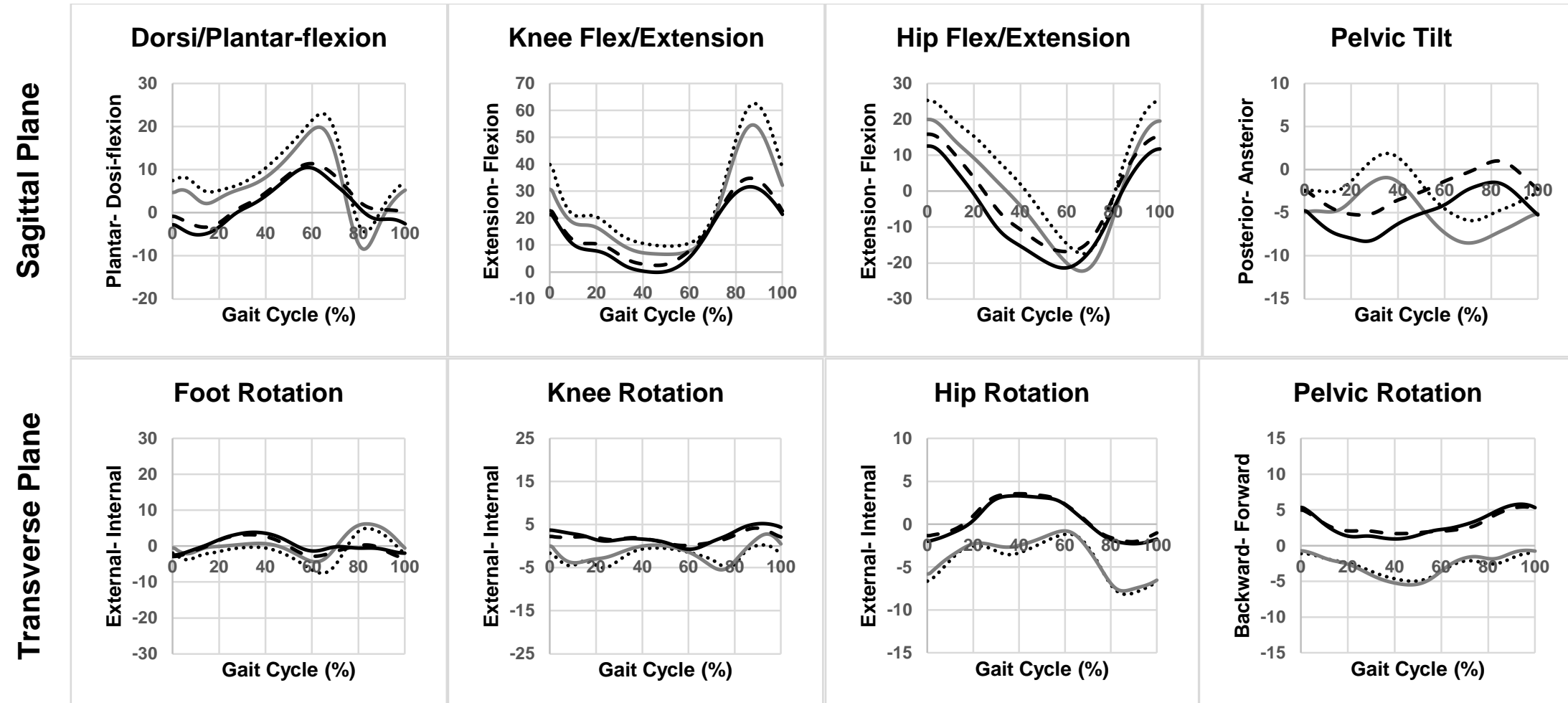

\section{Pelvic Rotation}

512
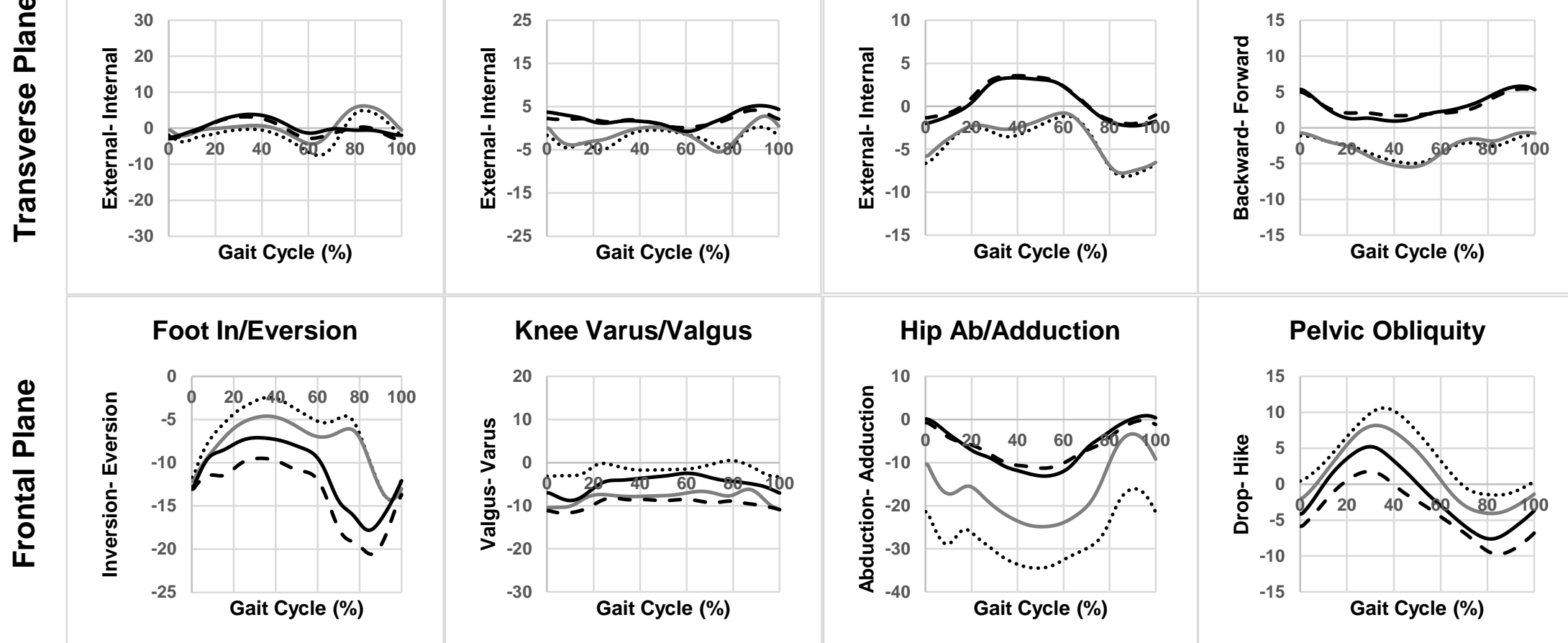

\section{Pelvic Obliquity}

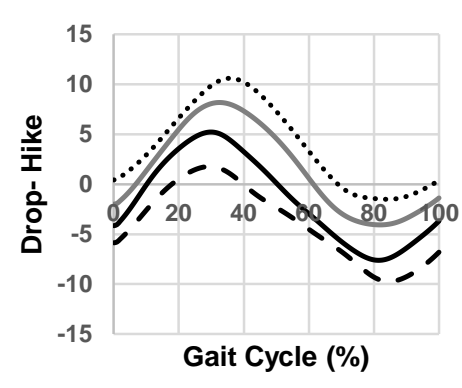

514 Figure 3. Three-dimensional kinetic data during walking with and without biofeedback system turned-on

- - - Affected side-Without biofeedback 

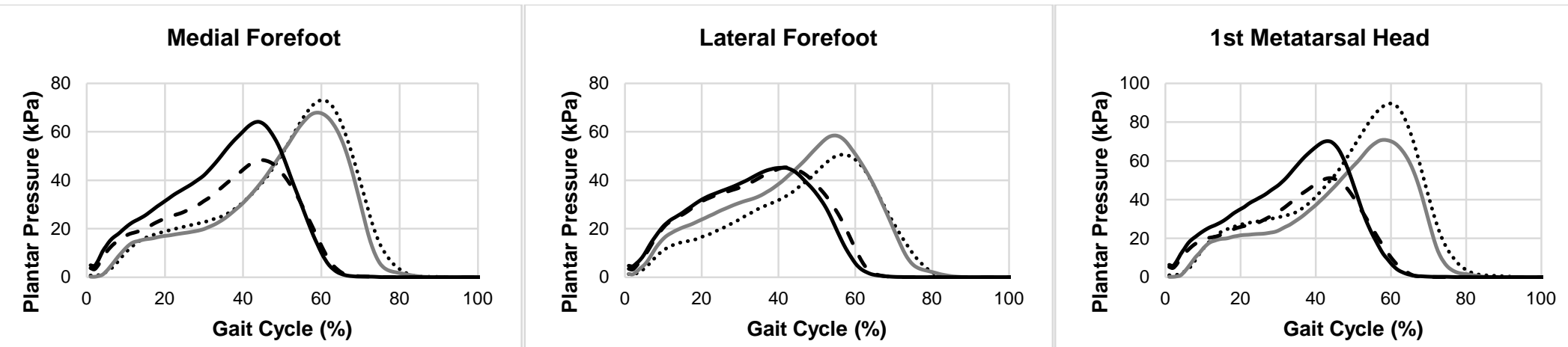

515
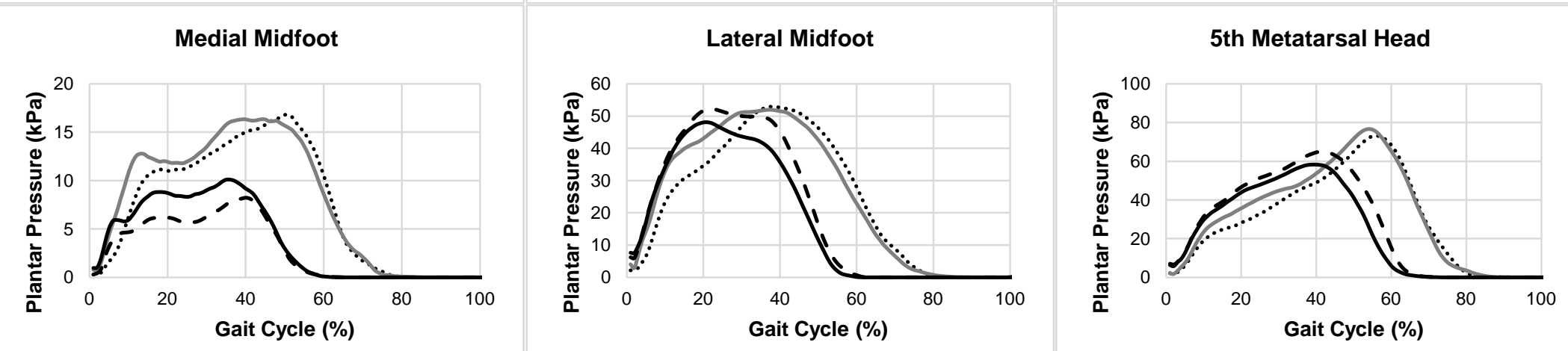

516
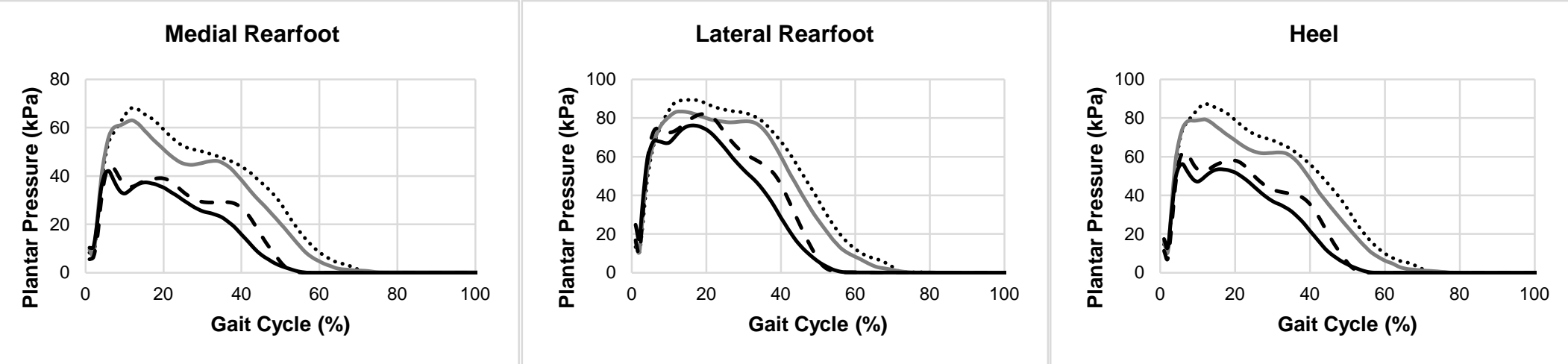

\section{Figure 4. Regional plantar pressure pattern in patients with and without biofeedback system turned-on}

- - - Affected side-Without biofeedback

- Affected side-With biofeedback 


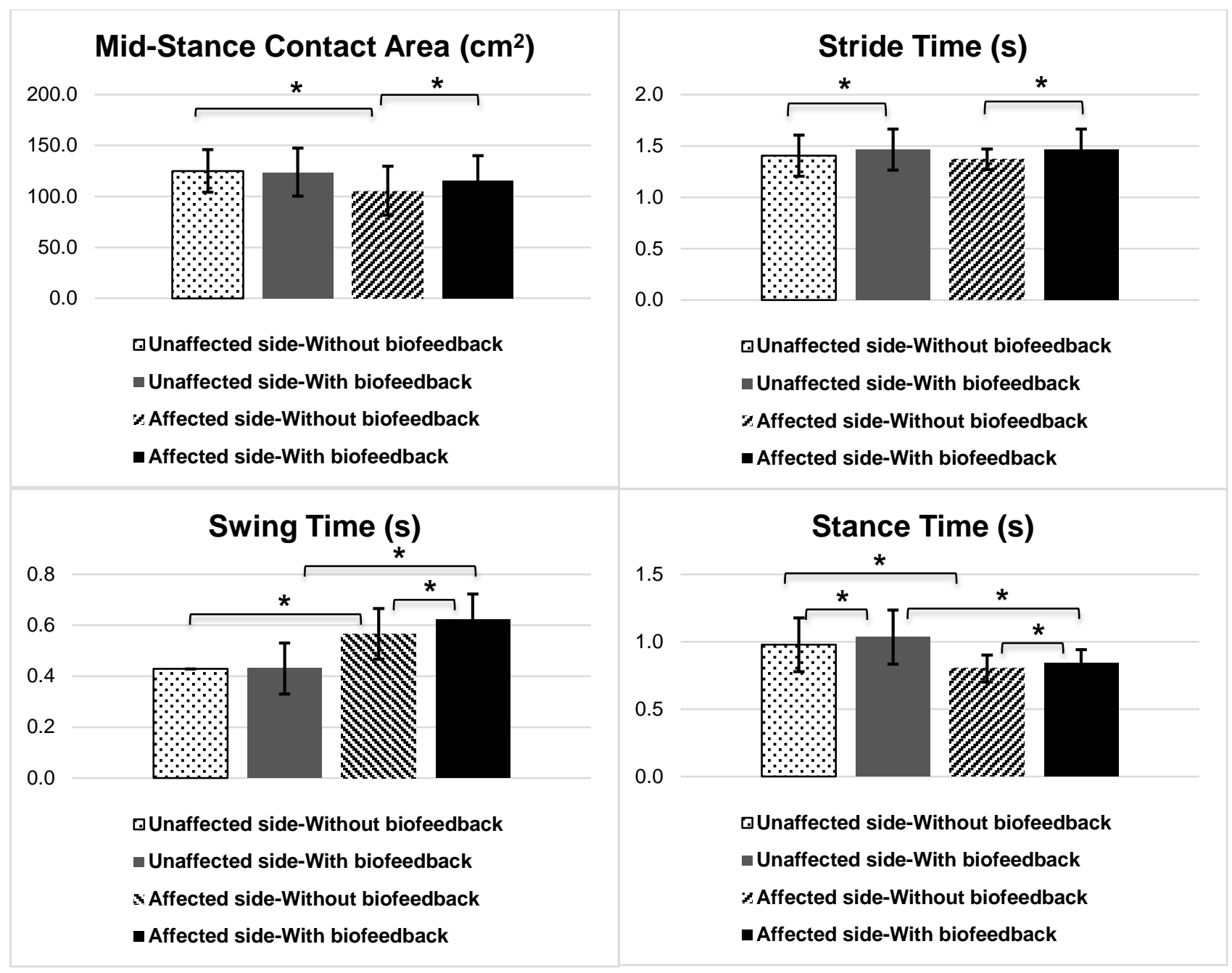

521 Figure 5. Contact area and temporal gait parameters in patients with and

522 without biofeedback system turned-on

$523 \quad *$ : Significant difference existed. 


\section{Figure captions}

526 Figure 1. The vibrotactile system, consisted of a plantar force acquisition unit and a vibrotactile 527 feedback unit wirelessly connected.

528 Figure 2. Foot regions: medial forefoot, lateral forefoot, medial midfoot, lateral midfoot, medial 529 rearfoot, and lateral rearfoot.

530 Figure 3. Three-dimensional kinetic data during walking with and without biofeedback system 531 turned-on.

532 Figure 4. Regional plantar pressure pattern in patients with and without biofeedback system turned533 on.

534 Figure 5. Contact area and temporal gait parameters in patients with and without biofeedback system 535 turned-on.

536 